Johann F. Castañeda, MD Jeffrey S. Concepcion, MD Ricardo L. Ramirez Jr., MD Kirt Areis Delovino, MD

Department Of Otorhinolaryngology Head and Neck Surgery St. Luke's Medical Center
Correspondence: Johann F. Castañeda, MD Department of Otorhinolaryngology Head and Neck Surgery St. Luke's Medical Center

279 E Rodriguez Sr. Ave., Quezon City 1102 Philippines

Telefax (632) 7230101 local 5543

Email:jong_castaneda@yahoo.com

Reprints will not be available from the author.

\section{Inflammatory Pseudotumor of the Maxillary Sinus}

Inflammatory pseudotumor (IPT) is a rarely occurring lesion with no identifiable local or systemic cause. First described in 1905 by Birch-Hirschfield, ${ }^{1}$ it remains somewhat of an enigmatic disease entity despite multiple otolaryngologic, radiologic and pathologic reports. The term "pseudotumor" was used because these lesions mimic invasive malignant tumors both clinically and radiologically. IPT most commonly involves the lung and orbit but has also been reported to occur at sites that make biopsy or excision difficult or potentially disfiguring. ${ }^{2}$ Its diagnosis and prompt recognition may help avoid radical surgery for this benign lesion.

\section{CASE}

A 27-year-old male was seen at our outpatient department due to a progressively enlarging left infraorbital mass. Two years prior, the patient noted a swelling over his left infraorbital area. The swelling was somewhat painful and rapidly grew in size so that it measured almost $2.5 \times 2.5 \mathrm{~cm}$ after a week. Still tender, it became firm and violaceous in color. He sought medical attention at a local hospital after one more week of persistent swelling and increasing cheek pain but denied excessive lacrimation, blurring of vision, orbital pain, eye discharge or numbness.

Incision and drainage of the left infraorbital mass yielded purulent material with resolution of the swelling and associated symptoms but a pea-sized mass was still palpable over the post operative site. Over the months that followed, the mass gradually increased in size with occasional serosanguinous discharge from the incision site. There was no pain, numbness or blurring of vision. He self-medicated with Cefalexin, taken irregularly for 8 months without any improvement before finally consulting again.

An orbital CT scan requested by the referring Ophthalmology service showed an expansile, mildly enhancing soft tissue mass with few peripheral foci of calcifications measuring $8.2 \times 4.4 \times 6.4 \mathrm{~cm}$ (Figures $1 \mathrm{~A}, B$ ) completely occupying the left maxillary sinus and extending up to the infero-lateral aspect of the left orbital cavity. There was erosion of the lateral portion of the left orbital floor and disruption of the frontal process of the left zygomatic bone with obliteration and effacement of the left pterygopalatine fossa. Our physical examination revealed a firm, fixed, nontender $4 \times 4 \mathrm{~cm}$ left inferior orbital mass with serosanguinous discharge and a bulging lateral nasal wall. Epiphora from the left eye suggested nasolacrimal duct obstruction but vision and extraocular movements were intact.

Caldwell-Luc biopsy surprisingly yielded only necrotic and inflammatory tissues despite generous samples from multiple sections of the maxillary portion and inflammatory polyps from the intranasal component. At surgery after a few weeks, the mass still occupied the entire left maxillary sinus despite the previous biopsy which had removed a significant amount of tumor. Furthermore, the mass now extended beyond the maxillary sinus into the left upper gingivobuccal area through the previous maxillary window. The entire clinically aggressive maxillary sinus mass was removed under endoscopic guidance but the final histopathology report was still similar to the 


\section{FEATURED GRAND ROUNDS}

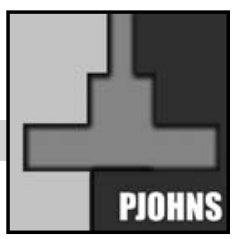

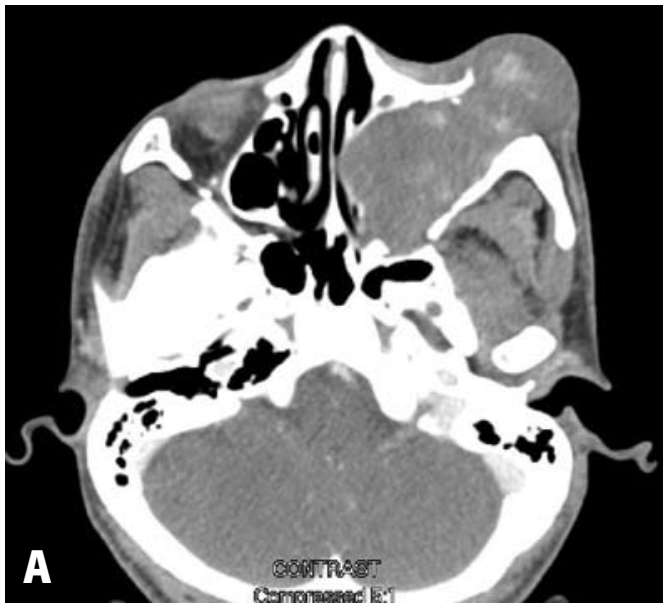

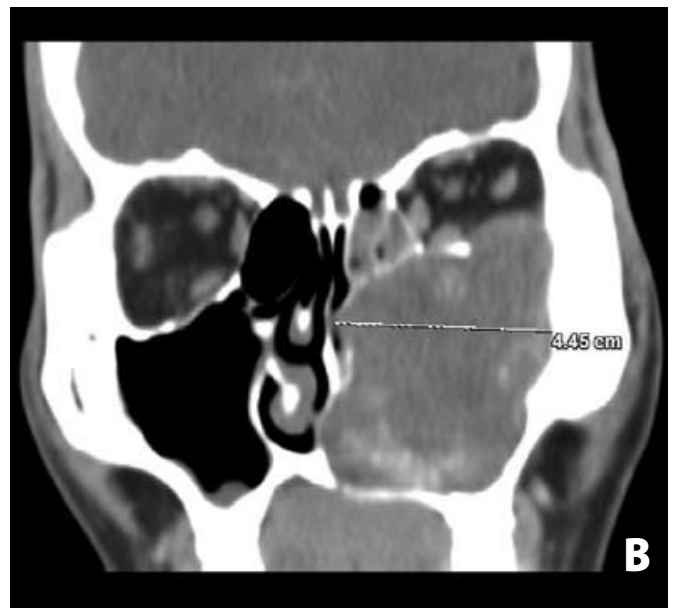

Figure 1 (A, Axial view; B, Coronal view) Contrast paranasa sinus CT scan showing expansile, mildly enhancing soft tissue mass with few peripheral foci of calcifications measuring $8.2 \times 4.4 \times 6.4 \mathrm{~cm}$ completely occupying the left maxillary sinus and extending up to the infero-lateral aspect of the left orbital cavity.

previous findings of necrotic and inflammatory tissues.

A month after surgery, the patient was seen at the Emergency room for left infraorbital swelling and discharge. Contrastenhanced MRI of the nasopharynx showed a large expansile left maxillary sinus lesion bulging into the nasal cavity, extending into adjacent lateral orbital soft tissue and extending into the buccal space through an apparently disrupted left inferolateral maxillary wall. Intravenous antibiotics and a high-dose steroid trial resulted in complete disappearance of the left infraorbital mass and discharge within a week and the patient was discharged on a tapering steroid dose.

\section{DISCUSSION}

Inflammatory pseudotumor is a quasi-neoplastic lesion that has been reported to occur in nearly every site in the body, most commonly involving the lung and the orbit, and rarely the maxillary sinus.' Its diagnosis is usually by exemption since clinical and histopathologic findings are sometimes vague and inconsistent. The exact etiology of these lesions is not clear. It has been postulated that they might be the result of a postinflammatory repair process, a metabolic disturbance or an antigen-antibody interaction with an agent that was no longer identifiable in aspiration or biopsy material. ${ }^{3}$ The clinical findings in a patient with an inflammatory pseudotumor are variable depending on the growth rate of the lesion and the specific structures that have been affected. Inflammatory pseudotumors have been reported to cause chronic cough (as a result of endobronchial growth), dry cough, fever, pleuritic pain, right upper quadrant or epigastric pain and several constitutional symptoms such as malaise, weight loss, fatigue and syncope. Inflammatory pseudotumors have been found incidentally during imaging examinations for other reasons. ${ }^{3}$

Extraorbital inflammatory pseudotumor of head and neck can occur in the nasal cavity, nasopharynx, maxillary sinus, larynx and trachea. Perineural spread along maxillary, mandibular and hypoglossal nerves had been described. Sinonasal inflammatory psuedotumors do not affect a particular age group and cause no systemic symptoms. However, they have a more aggressive appearance than those of the orbit with bony changes such as erosion, remodelling and sclerosis usually seen on radiographic studies. ${ }^{4}$ On CAT scans, a moderately enhancing soft tissue mass is usually seen accompanied by bony changes common among malignant processes. ${ }^{5}$ On cut sections, inflammatory cells dominate as well as necrotic tissues. In some patients, laboratory findings are normal; in others, there might be an elevated erythrocyte sedimentation rate and C-reactive protein level and sometimes a high white blood cell count. ${ }^{3}$ However, none of the published reports on inflammatory pseudotumor have mentioned any presence of positive tumor markers. Complete surgical resection if possible is the treatment of choice for sinonasal inflammatory pseudotumors, followed by corticosteroids in cases of incomplete excision. Response to steroids is often unpredictable but these drugs are the primary treatment method for orbital inflammatory pseudotumor. The only cases in which radiation therapy is indicated are those patients for whom surgery or corticosteroid therapy is unsuccessful or contraindicated. ${ }^{6}$

\footnotetext{
REFERENCES

1. Agarwal A. Orbital Pseudotumor; Diagnosis on Fine Needle Aspiration Cytology. J Cytol 2008 Apr; 25(2):67-69.

2. Narla LD, Newman B, Spottswood SS, Narla S, Kolli R. Inflammatory pseudotumor. Radiographics 2003 May-Jun; 23(3): 719-729.

3. Restrepo S, Mastrogiovanni LP, Palacios E. Inflammatory pseudotumor of the trachea. Ear Nose Throat J. 2003 Jul; 82(7): 510-2.

4. De Vuysere S, Hermans R, Sciot R, Crevits I, Marchal G. Extraorbital Inflammatory Pseudotumor of the Head and Neck: CT and MR findings in three patients. Am J Neuroradiol 1999; 20:1133-1134.

5. Park SB. Lee JH, Weon YC. Imaging findings of head and neck inflammatory pseudotumor. Am J Roentgenol. 2009 Oct; 193(4): 1180-1186.

6. Ruaux C, Noret P, Godey B. Inflammatory Pseudotumor of the nasal cavity and sinuses. Laryngol Otol. 2001; 115:563-566.
} 